\title{
Assessment of liver injury using indocyanine green fluorescence imaging
}

\author{
Yan Chen ${ }^{1}$, Ge Liu ${ }^{1}$, Yulin $\mathrm{Wu}^{2}$, Huiming $\mathrm{Cai}^{2}$ \\ ${ }^{1}$ School of Basic Medicine and Clinical Pharmacy, China Pharmaceutical University, Nanjing, China; ${ }^{2}$ Nanjing Nuoyuan Medical Devices Co., Ltd., \\ Nanjing, China \\ Contributions: (I) Conception and design: Y Chen, H Cai; (II) Administrative support: H Cai; (III) Provision of study materials or patients: Y Wu; (IV) \\ Collection and assembly of data: Y Chen; (V) Data analysis and interpretation: G Liu; (VI) Manuscript writing: All authors; (VII) Final approval of \\ manuscript: All authors. \\ Correspondence to: Huiming Cai. Fuqian South Road 18, Nanjing 211514, China. Email: chm9166@aliyun.com.
}

\begin{abstract}
Background: To investigate whether indocyanine green (ICG) fluorescence imaging can be used to evaluate chronic and acute liver injury induced by either a high-fat (HF) diet or carbon tetrachloride $\left(\mathrm{CCl}_{4}\right)$.

Methods: Sprague-Dawley (SD) rats were randomly divided into three groups: control group, HF diet-induced model group, and $\mathrm{CCl}_{4}$-induced model group. The chronic and acute liver injury models were induced by a $\mathrm{HF}$ diet and intraperitoneal injection of $\mathrm{CCl}_{4}$, respectively. After $\mathrm{HF}$ feeding, the liver index, levels of serum alanine aminotransferase (ALT) and aspartate aminotransferase (AST) of the rats were determined. The livers were also collected to evaluate histopathology damage by hematoxylin and eosin (H\&E) staining. After in vitro perfusion of the liver and ICG administration, the liver fluorescence intensity and corresponding spectral value were measured by using real-image guided system (REAL-IGS).

Results: After HF feeding, the liver index and levels of serum ALT and AST were significantly increased, and the livers of the rats showed severe histopathological changes. Compared with the control group, the hepatic lobes of the model rats exhibited incomplete green fluorescence, and the corresponding spectral value was markedly reduced.

Conclusions: ICG fluorescence imaging can be used to evaluate liver injury induced by either a HF diet or $\mathrm{CCl}_{4}$.
\end{abstract}

Keywords: Liver injury; indocyanine green (ICG); fluorescence imaging

Submitted May 25, 2021. Accepted for publication Jul 14, 2021.

doi: 10.21037/atm-21-3049

View this article at: https://dx.doi.org/10.21037/atm-21-3049

\section{Introduction}

The liver plays an essential role in various metabolic processes, including glucose homeostasis and detoxification, proteins synthesis, as well as the utilization of various nutrients (1). Generally, liver injury is considered a result of exposure to high levels of toxic chemicals and drugs, which are associated with metabolic dysfunctions, ranging from the transient elevation of liver enzymes to life-threatening liver cirrhosis, hepatic fibrosis, and even hepatocellular carcinoma (2). The pathogenic factors of liver injury are complex, accompanied by a rapid decline in liver function during the period, and even death in severe cases (3).
Among them, fatty liver and carbon tetrachloride $\left(\mathrm{CCl}_{4}\right)^{-}$ induced acute liver injury can cause liver microcirculation damage and reduction of effective blood flow due to fat accumulation or collagen fiber hyperplasia (4).

At present, optical molecular imaging technology is developing rapidly, and offers advantages such as being real-time, non-invasive, precise, and sensitive (5). Among them, indocyanine green (ICG) is commonly used as a fluorescent dye, which is completely absorbed by the liver through a selective mechanism and metabolized with bile in an unbound state. It can be used to detect the blood flow of the liver to reflect the active state of liver cells 
$(6,7)$. This technology has been applied in the resection of centrally located liver tumors and hilar (Klatskin's) tumors (8). There are few studies regarding its application in other liver diseases. In this paper, we hypothesized that ICG fluorescence imaging technology could be applied to evaluate liver pathological damage caused by high-fat (HF) feeding and $\mathrm{CCl}_{4}$. The study may provide preclinical evidence for us to develop fluorescent imaging techniques for the evaluation of liver function in the future. We present the following article in accordance with the ARRIVE reporting checklist (available at https://dx.doi.org/10.21037/ atm-21-3049).

\section{Methods}

\section{Animals and treatment}

Male Sprague-Dawley (SD) rats (180-200 g) were purchased from Qinglongshan Animal Breeding Centre (Nanjing, China). Rats were fed freely in a temperaturecontrolled room $\left(22 \pm 2{ }^{\circ} \mathrm{C}\right)$ with a 12 h light-dark cycle. To induce acute liver injury, the rats received a $50 \% \mathrm{CCl}_{4}$ solution ( $1 \mathrm{~mL} / \mathrm{kg}$, dissolved in corn oil) intraperitoneally, as previously described (9). The chronic liver injury model was induced by HF feed (formulation: $2 \%$ cholesterol, $0.5 \%$ bile salt, $12 \%$ lard, $0.2 \%$ propyl, and the remaining $85.3 \%$ as basic feed) for 10 weeks. Control rats were fed normally and injected with the same volume of the corn oil vehicle intraperitoneally. For evaluation of blood biochemistry, near-infrared fluorescence imaging and histopathological staining were utilized ( $\mathrm{n}=5$ for each group). All experimental procedures were performed under a project license (No.: 2021-06-012) granted by institutional ethics board of China Pharmaceutical University (Nanjing, China), in compliance with the National Institutes of Health (Bethesda, MD, USA) guidelines for the care and use of animals.

\section{Blood biochemistry}

The rats were anesthetized by $3 \%$ chloral hydrate $(300 \mathrm{mg} / \mathrm{kg}$, i.p), and blood was collected from the fundus venous plexus. The serum was separated by centrifugation at 3,000 rpm for $10 \mathrm{~min}$ at $4^{\circ} \mathrm{C}$. The levels of serum triglyceride (TG), total cholesterol (TC), alanine aminotransferase (ALT), and aspartate aminotransferase (AST) were measured using test kits (Nanjing Jiancheng Institute of Biological Engineering, Nanjing, China) according to the manufacturer's instructions.

\section{Near-infrared fuorescence imaging}

The rats were sacrificed and their livers were collected and weighed before extracorporeal perfusion with Perfusate (Zhongyi Medical Technology, Shanghai, China). The livers were then injected with a different concentration of ICG solution $(1 \mathrm{~mL} / 10 \mathrm{~g}$, Dandong Yichuang Pharmaceutical Co., Ltd., Dangdong, China) through the portal vein, and detected under a near-infrared fluorescence imaging system (REAL-IGS, Nanjing Shengshengyuan Pharmaceutical Industry Co., Ltd., Nanjing, China). The images of the livers and the corresponding spectral values were recorded after 15 minutes of ICG administration.

\section{Histopatbological staining}

For the histological investigations, liver tissues were removed from a portion of the left lobe, and cryosections were cut at a thickness of $6 \mu \mathrm{m}$. After hematoxylin and eosin (H\&E) staining, the slides were observed for conventional morphological evaluation under a light microscope (Olympus BX53, Japan) and photographed at 200x magnification. The histological changes were scored according to previous reports (10).

* "0": normal hepatic cell structure;

* "1": fatty changes of hepatocytes $(<1 / 3)$, scattered inflammatory cells, and ballooning degeneration of hepatic cells;

* "2": fatty changes of hepatocytes (approximately $1 / 3-2 / 3)$, focal inflammatory cell infiltration, and severe ballooning degeneration of hepatic cells;

* "3": extensive hepatocellular fatty changes $(>2 / 3)$, and patchy inflammatory cell infiltration.

\section{Statistical analysis}

Statistical analysis was performed by IBM SPSS Statistics v22.0 software (USA), and the data were presented as means \pm standard deviation (SD). One-way analysis of variance (ANOVA) was used to compare the statistical differences between groups. Differences were considered statistically significant at $\mathrm{P}<0.05$. Statistical figures were constructed using Adobe Photoshop CC 2019 (USA) and GraphPad Prism Version 8.0 (USA).

\section{Results}

As shown in Figure 1, near-infrared fluorescence images of 

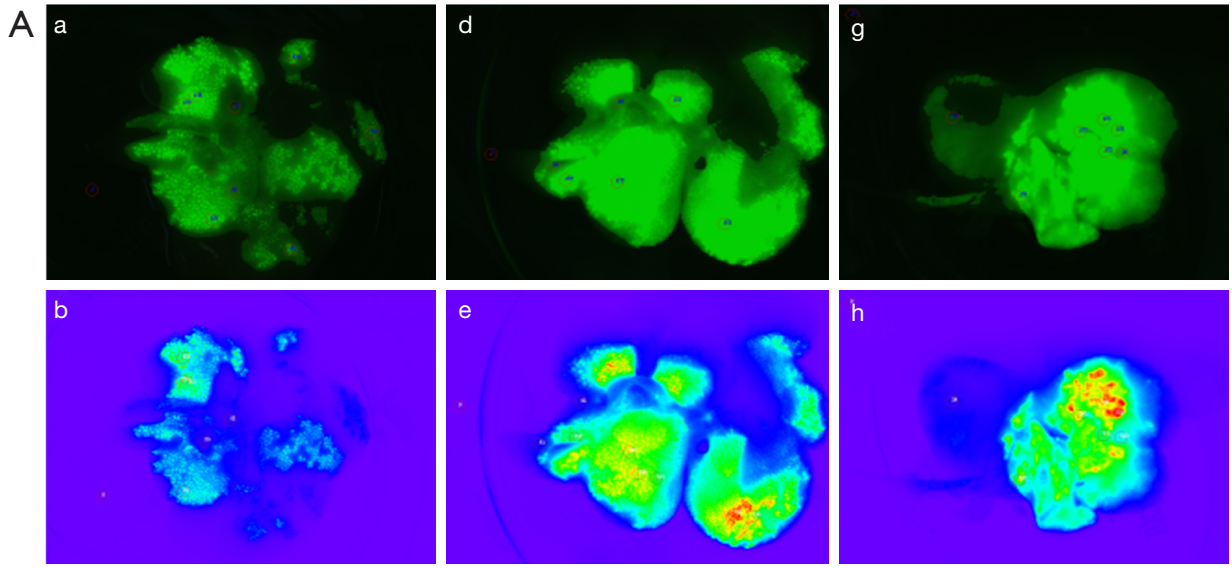

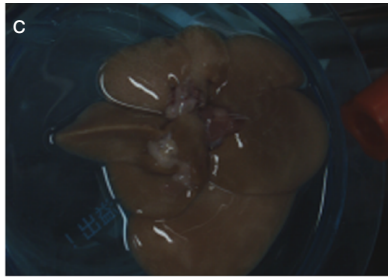

$0.01 \mathrm{mg} / \mathrm{mL}$

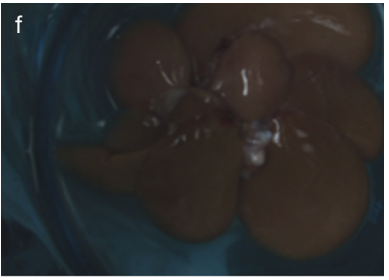

$0.02 \mathrm{mg} / \mathrm{mL}$

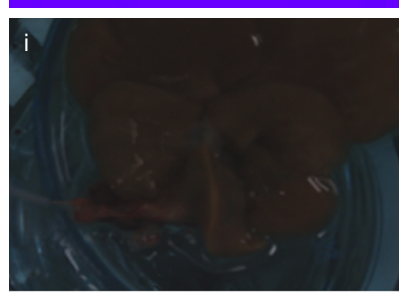

$0.05 \mathrm{mg} / \mathrm{mL}$

B

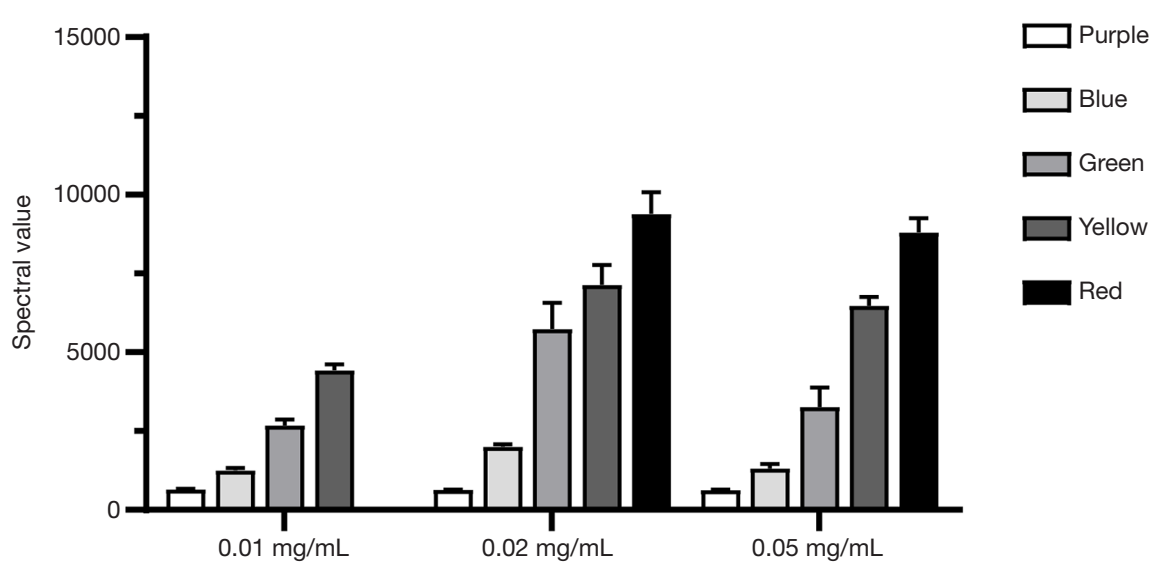

Figure 1 Imaging effect of normal liver after ICG injection. (A) Different images of normal liver under (a, d, g) near-infrared fluorescence, (b, e, h) fusion pseudo light, and (c, f, i) visible light. (B) Corresponding spectral values of different intensities in near-infrared fluorescence images. Values were shown as mean $\pm \mathrm{SD}(\mathrm{n}=5)$. The different colors in the figure represent different fluorescence intensities. ICG, indocyanine green.

control livers displayed all colors (purple to red) after ICG injection at concentrations of 0.02 and $0.05 \mathrm{mg} / \mathrm{mL}$. When compared with $0.05 \mathrm{mg} / \mathrm{mL}$, the spectral values of different fluorescence intensities at a concentration of $0.02 \mathrm{mg} / \mathrm{mL}$ were higher, and thus, $0.02 \mathrm{mg} / \mathrm{mL}$ of ICG was selected to inject through the portal vein after in vitro perfusion.

As shown in Figure $2 A$, the results of $\mathrm{H} \& \mathrm{E}$ staining showed that HF feed induced harmful effects on the rat livers. HF feed caused visible histological changes, including extensive inflammatory infiltration and ballooning degeneration of hepatic cells in the liver. Moreover, the liver injury score significantly increased in the HF model rats as compared with the controls $(\mathrm{P}<0.01$, Figure $2 B)$. Furthermore, compared with the control group, the ratio 
A

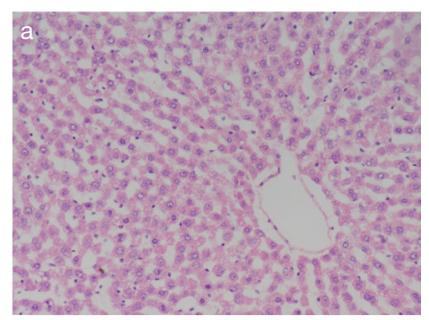

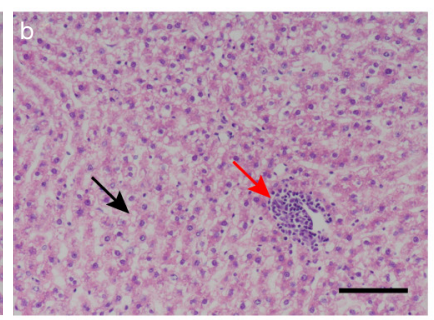

D

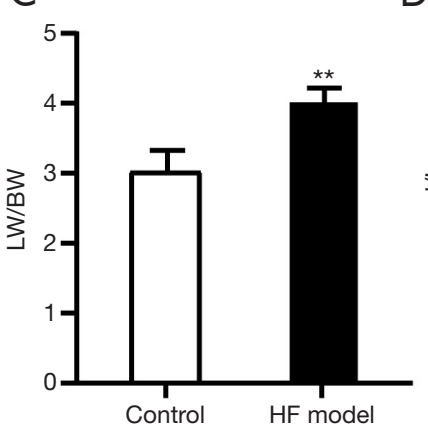

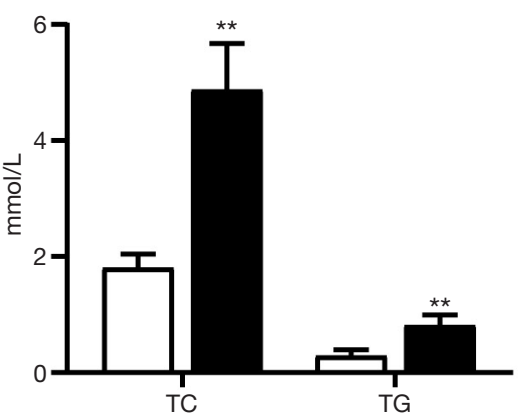

B

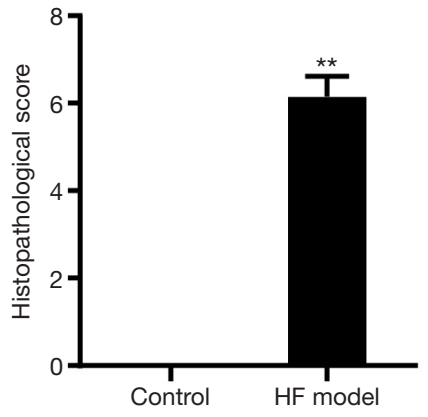

$\mathrm{E}$

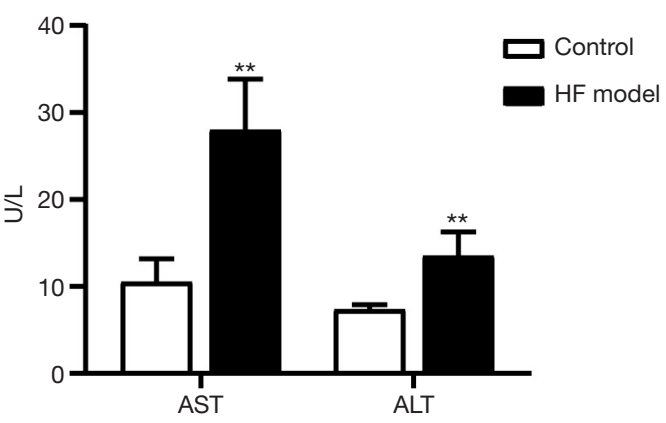

Figure 2 Changes of liver histomorphology and function in CCl4-treated mice. (A) H\&E staining shows the extensive cellular damage and infiltration of inflammatory cells in (a) control rats and (b) HF model rats. The black arrow indicates ballooning degeneration. The red arrow indicates infiltrating inflammatory cells. Scale bars, $100 \mu \mathrm{m}$. (B) Qualification of hepatic histological injury. (C) Relative LW/BW of rats. (D) The levels of serum TC and TG in rats. (E) The levels of serum AST and ALT in rats. Values were shown as mean \pm SD $(n=5)$. ${ }^{* *} \mathrm{P}<0.01$, vs. control group. H\&E, hematoxylin and eosin; HF, high-fat; LW/BW, liver weight versus body weight; TC, total cholesterol; TG, triglyceride; AST, aspartate aminotransferase; ALT, alanine aminotransferase.

of liver weight versus body weight (LW/BW) enhanced in the HF model rats $(\mathrm{P}<0.01$, Figure $2 C)$. HF feed also significantly increased the levels of serum TC, TG, AST, and ALT $(\mathrm{P}<0.01$, Figure 2D,E). These results indicated that 10 weeks of $\mathrm{HF}$ feed induced severe liver injury.

ICG fluorescence imaging was performed to evaluate liver damage after ICG injection (Figure $3 A$ ). The control group displayed intact liver lobes under near-infrared fluorescence and fusion pseudo light, while the HF model group and $\mathrm{CCl}_{4}$ model group exhibited incomplete liver lobes. As shown in Figure 3B, when the fluorescence intensity was shown as yellow and green, the liver spectrum values of $\mathrm{HF}$ model group and $\mathrm{CCl}_{4}$ model group significantly decreased as compared with the control group $(\mathrm{P}<0.01)$.

\section{Discussion}

In this experiment, a HF diet and intraperitoneal injection of $\mathrm{CCl}_{4}$ were used to induce chronic and acute liver injury in rats, respectively. After HF feeding, the liver index and levels of serum TC, TG, AST, and ALT increased significantly. Moreover, the H\&E staining results showed that the livers of HF model rats displayed extensive inflammatory infiltration and ballooning degeneration of hepatic cells. These results indicated that the liver injury model had been successfully established. The liver of each rat was administered the same concentration of ICG solution after in vitro perfusion. After ICG fluorescence imaging, the $\mathrm{HF}$ and $\mathrm{CCl}_{4}$ model groups displayed incomplete liver lobes under near-infrared fluorescence and fusion pseudo light. Meanwhile, when the fluorescence intensity was yellow and green, the liver spectrum values of the $\mathrm{CCl}_{4}$ model group and the HF model group were significantly reduced, indicating that ICG fluorescence imaging can be used to evaluate rats with HF diet- and $\mathrm{CCl}_{4}$-induced liver injury.

ICG is a water-soluble tricarbocyanine dye developed by 


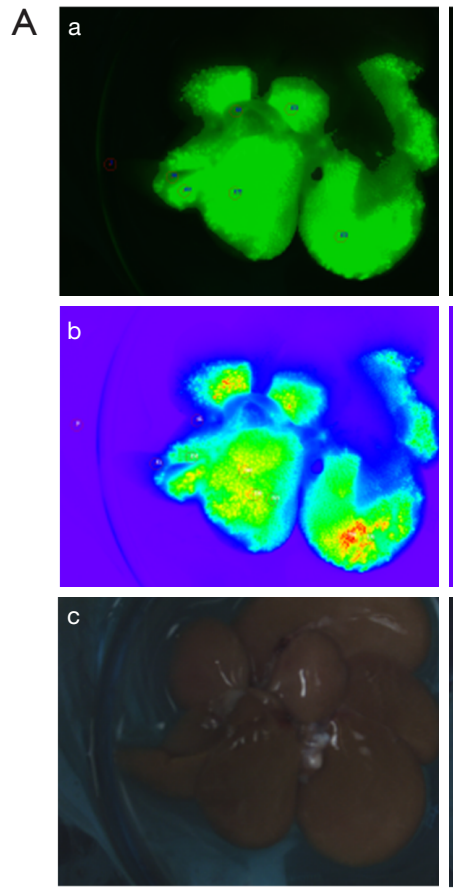

Control
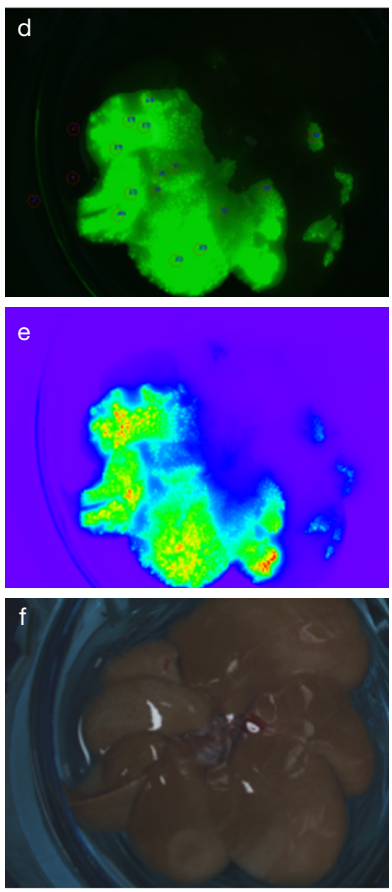

HF model
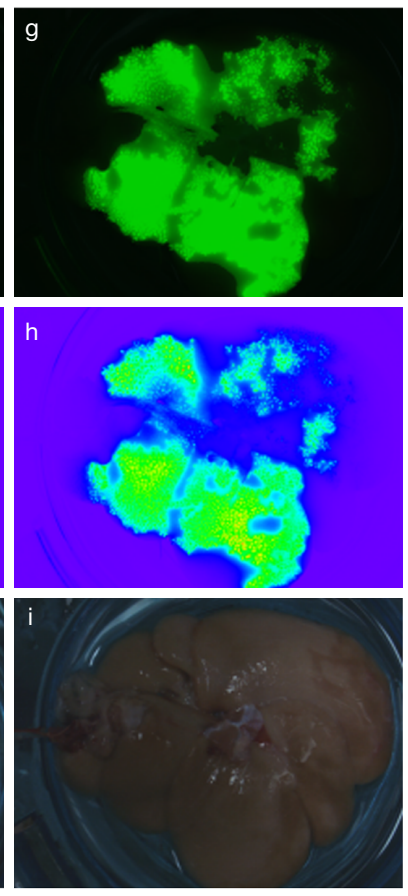

$\mathrm{CCL}_{4}$ model

B

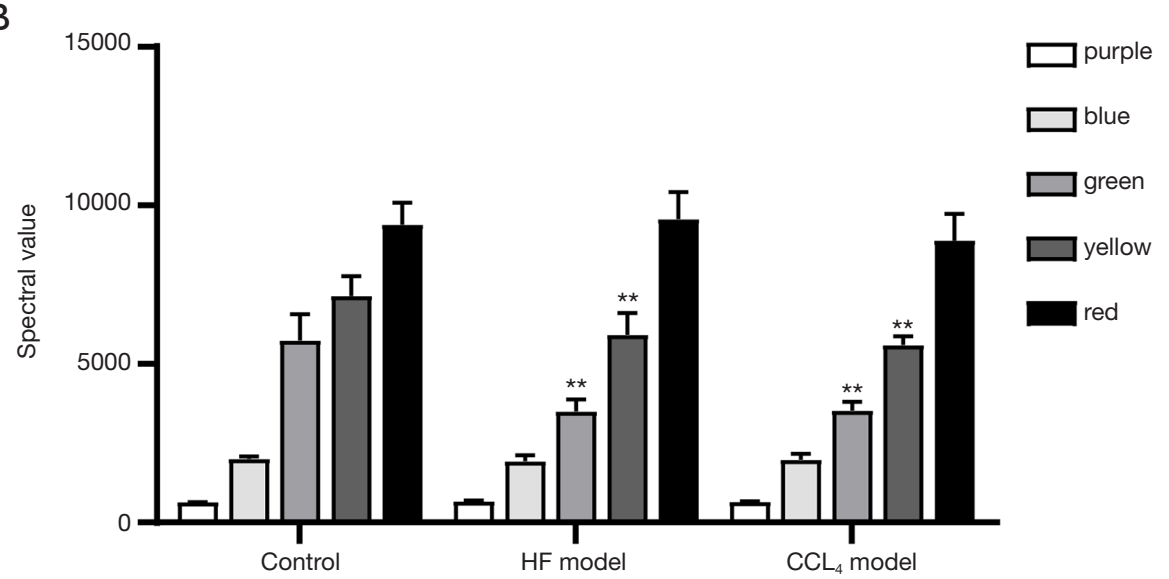

Figure 3 Imaging effect of normal liver after ICG injection. (A) Different images of normal liver under (a, d, g) near-infrared fluorescence, (b, e, h) fusion pseudo light, and (c, f, i) visible light. (B) Corresponding spectral values of different intensities in near-infrared fluorescence images. Values were shown as mean $\pm \mathrm{SD}(\mathrm{n}=5)$. ${ }^{* *} \mathrm{P}<0.01$, vs. control group. ICG, indocyanine green.

Kodak Research Laboratory in 1955. ICG can be activated by extraneous light with a wavelength of $750-810 \mathrm{~nm}$ to emit $840 \mathrm{~nm}$ near-infrared light. After being injected into the cycle, it combines with plasma proteins (albumin, $\alpha 1$ lipoprotein) completely and enters the liver. It is then taken up by hepatocytes and subsequently excreted into bile in an unbound state (7). ICG enters hepatocytes of normal liver tissue quickly and emits fluorescence, the intensity of which gradually weakens after excretion into bile (11). The detected fluorescence intensity of ICG is eliminated in a typical linear manner, which can be used to evaluate liver reserve function after intravenously injection at a high dose (12).

The entry of ICG into hepatocytes is blocked due to liver injury induced by ischemia or cirrhosis $(13,14)$. Previous studies have shown that ICG-mediated fluorescence imaging technology mainly focuses on real-time positioning of tumor tissue during surgery. This real-time, non-invasive, and sensitive technology can be used to detect tumor lesion 
boundaries, liver resection range, as well as small and residual lesions $(8,15,16)$. This technique is widely used in the diagnosis of liver tumors, however there are few studies regarding its application in other liver diseases.

The liver is a critical organ, where many physiological and biochemical processes occur in the human body, and is closely related to human health (1). The liver accounts for $2.5 \%$ of the total body weight and utilizes nearly $25 \%$ of the cardiac output (17). Fatty infiltration of liver cells leads to cell swelling and degeneration in fatty liver patients (18). At the same time, the resistance of hepatocytes' portal blood flow increases, causing liver microcirculation damage and effective blood flow reduction $(19,20)$. In this study, the liver cells of rats in $\mathrm{HF}$ and $\mathrm{CCl}_{4}$ model groups showed severe steatosis and mild necrosis, which reduced the intake of ICG by hepatocytes. Compared with the control group, the liver lobes of rats in the $\mathrm{HF}$ and $\mathrm{CCl}_{4}$ model groups under nearinfrared fluorescence displayed incomplete fluorescence, and the corresponding spectrum values were significantly reduced at the same fluorescence intensity. These results indicate that ICG fluorescence imaging can be used for visual evaluation of liver injury induced by $\mathrm{HF}$ feeding and $\mathrm{CCl}_{4}$ to a certain degree.

ICG is administered via the portal vein to detect the intake by the liver, which can directly reflect the perfusion of the liver to assess its function. Compared with the ICG plasma clearance test that is commonly used clinically, the injection of ICG after in vitro perfusion can avoid the disturbance of some factors, such as arteriovenous fistulas, bilirubin, portal vein cancer thrombi, vasodilators, bile excretion, and secretion disorders, which affect liver blood flow and lead to test result instability (21). In this study, the liver was perfused in vitro before the administration of ICG to eliminate the influence of blood flow. In the future, it can be applied to evaluate donation after cardiac death (DCD) donor livers. Importantly, it is necessary to perform pathological examination and reserve function evaluation of the donor liver before liver transplantation. Currently, the commonly used methods, such as liver biopsy, serum biochemical index examination and imaging examination $(22,23)$, are not fully applicable due to the diminishing accuracy of liver functional assessment $(24,25)$. Quantitative methods for liver function with high accuracy and practicability, such as the ICG clearance test $(26,27)$, can only be performed on live livers. Therefore, the development of more effective methods to assess DCD donor liver function may contribute to solving the current insufficiency of donors (28).
In summary, ICG can be injected through the portal vein after in vitro perfusion, resulting in a good fluorescence imaging effect. When the liver is damaged due to a HF diet and $\mathrm{CCl}_{4}$, the fluorescence intensity and corresponding spectral value under ICG fluorescence imaging can dynamically reflect the liver damage. This method is simple, practical, and safe, and may have application prospects in the field of liver transplantation.

\section{Acknowledgments}

Funding: This work was supported by the National Key Research and Development Program of China (2016YFC0104100) and the Key Promotion Project of Core Technology of Jiangsu province (2018-2020).

\section{Footnote}

Reporting Checklist: The authors have completed the ARRIVE reporting checklist. Available at https://dx.doi. org/10.21037/atm-21-3049

Data Sharing Statement: Available at https://dx.doi. org/10.21037/atm-21-3049

Conflicts of Interest: All authors have completed the ICMJE uniform disclosure form (available at https://dx.doi. org/10.21037/atm-21-3049). YW and HC are employees of Nanjing Nuoyuan Medical Devices Co., Ltd. The other authors have no conflicts of interest to declare.

Ethical Statement: The authors are accountable for all aspects of the work in ensuring that questions related to the accuracy or integrity of any part of the work are appropriately investigated and resolved. All experimental procedures were performed under a project license (No.: 2021-06-012) granted by institutional ethics board of China Pharmaceutical University (Nanjing, China), in compliance with the National Institutes of Health (Bethesda, MD, USA) guidelines for the care and use of animals.

Open Access Statement: This is an Open Access article distributed in accordance with the Creative Commons Attribution-NonCommercial-NoDerivs 4.0 International License (CC BY-NC-ND 4.0), which permits the noncommercial replication and distribution of the article with the strict proviso that no changes or edits are made and the original work is properly cited (including links to both the 
formal publication through the relevant DOI and the license). See: https://creativecommons.org/licenses/by-nc-nd/4.0/.

\section{References}

1. 1. Trefts E, Gannon M, Wasserman DH. The liver. Curr Biol 2017;27:R1147-51.

2. Sun $\mathrm{H}$, Chen $\mathrm{L}, \mathrm{Zhou} \mathrm{W}$, et al. The protective role of hydrogen-rich saline in experimental liver injury in mice. J Hepatol 2011;54:471-80.

3. Chiu YJ, Chou SC, Chiu CS, et al. Hepatoprotective effect of the ethanol extract of Polygonum orientale on carbon tetrachloride-induced acute liver injury in mice. J Food Drug Anal 2018;26:369-79.

4. Pasarín M, Abraldes JG, Liguori E, et al. Intrahepatic vascular changes in non-alcoholic fatty liver disease: Potential role of insulin-resistance and endothelial dysfunction. World J Gastroenterol 2017;23:6777-87.

5. Wang C, Wang Z, Zhao T, et al. Optical molecular imaging for tumor detection and image-guided surgery. Biomaterials 2018;157:62-75.

6. Wang Y, Zhang Y, Yu Y, et al. Preparation and preliminary evaluation of hepatitis B core antigen virus like nanoparticles loaded with indocyanine green. Ann Transl Med 2020;8:1661.

7. Sakka SG. Assessment of liver perfusion and function by indocyanine green in the perioperative setting and in critically ill patients. J Clin Monit Comput 2018;32:787-96.

8. Kobayashi Y, Kawaguchi Y, Kobayashi K, et al. Portal vein territory identification using indocyanine green fluorescence imaging: Technical details and short-term outcomes. J Surg Oncol 2017;116:921-31.

9. Khan A, Shal B, Naveed M, et al. Matrine ameliorates anxiety and depression-like behaviour by targeting hyperammonemia-induced neuroinflammation and oxidative stress in $\mathrm{CCl} 4$ model of liver injury. Neurotoxicology 2019;72:38-50.

10. Wang F, Xue Y, Yang J, et al. Hepatoprotective effect of apple polyphenols against concanavalin A-induced immunological liver injury in mice. Chem Biol Interact 2016;258:159-65.

11. Zhang $\mathrm{P}, \mathrm{Luo} \mathrm{H}, \mathrm{Zhu} \mathrm{W}$, et al. Real-time navigation for laparoscopic hepatectomy using image fusion of preoperative $3 \mathrm{D}$ surgical plan and intraoperative indocyanine green fluorescence imaging. Surg Endosc 2020;34:3449-59.

12. Zhang Y, Zhang L, Yin G, et al. Assessing indocyanine green pharmacokinetics in mouse liver with a dynamic diffuse fluorescence tomography system. J Biophotonics 2018;11:e201800041.

13. Levesque E, Martin E, Dudau D, et al. Current use and perspective of indocyanine green clearance in liver diseases. Anaesth Crit Care Pain Med 2016;35:49-57.

14. Nowak K, Sandra-Petrescu F, Post S, et al. Ischemic and injured bowel evaluation by Fluorescence imaging. Colorectal Dis 2015;17 Suppl 3:12-5.

15. Nishino H, Hatano E, Seo S, et al. Real-time Navigation for Liver Surgery Using Projection Mapping With Indocyanine Green Fluorescence: Development of the Novel Medical Imaging Projection System. Ann Surg 2018;267:1134-40.

16. Gotoh K, Yamada T, Ishikawa O, et al. A novel imageguided surgery of hepatocellular carcinoma by indocyanine green fluorescence imaging navigation. J Surg Oncol 2009;100:75-9.

17. Eipel C, Abshagen K, Vollmar B. Regulation of hepatic blood flow: the hepatic arterial buffer response revisited. World J Gastroenterol 2010;16:6046-57.

18. Jakovljevic DG, Hallsworth K, Zalewski P, et al. Resistance exercise improves autonomic regulation at rest and haemodynamic response to exercise in non-alcoholic fatty liver disease. Clin Sci (Lond) 2013;125:143-9.

19. Liu Y, Wen PH, Zhang XX, et al. Breviscapine ameliorates CCl4-induced liver injury in mice through inhibiting inflammatory apoptotic response and ROS generation. Int J Mol Med 2018;42:755-68.

20. Hafez MM, Al-Harbi NO, Al-Hoshani AR, et al. Hepatoprotective effect of rutin via IL-6/STAT3 pathway in CCl4-induced hepatotoxicity in rats. Biol Res 2015;48:30.

21. Jiao LR, El-Desoky AA, Seifalian AM, et al. Effect of liver blood flow and function on hepatic indocyanine green clearance measured directly in a cirrhotic animal model. $\mathrm{Br}$ J Surg 2000;87:568-74.

22. Li B, Min J, Liang WR, et al. Use of magnetic resonance elastography for assessing liver functional reserve: A clinical study. World J Gastroenterol 2015;21:7522-8.

23. Zenitani M, Hosoda H, Kodama T, et al. Postoperative decrease in plasma acyl ghrelin levels after pediatric living donor liver transplantation in association with hepatic damage due to ischemia and reperfusion injury. Pediatr Surg Int 2019;35:709-14.

24. Bedossa P, Poitou C, Veyrie N, et al. Histopathological algorithm and scoring system for evaluation of liver lesions in morbidly obese patients. Hepatology 2012;56:1751-9.

25. Lee S, Kim KW, Lee J, et al. Portal Vein Flow by Doppler 
Page 8 of 8

Ultrasonography and Liver Volume by Computed Tomography in Living Donor Candidates: Correlation with Indocyanine Green Test. Exp Clin Transplant 2019;17:627-31.

26. Xiang Y, Song F, Yang XH, et al. Evaluation of the significance of hepatic functional reserve for the operation of liver cancer complicated with cirrhosis. J BUON 2017;22:932-5.

Cite this article as: Chen Y, Liu G, Wu Y, Cai H. Assessment of liver injury using indocyanine green fluorescence imaging. Ann Transl Med 2021;9(14):1167. doi: 10.21037/atm-21-3049
27. Mándli T, Fazakas J, Ther G, et al. Evaluation of liver function before living donor liver transplantation and liver resection. Orv Hetil 2008;149:779-86.

28. Doyle MB, Collins K, Vachharajani N, et al. Outcomes Using Grafts from Donors after Cardiac Death. J Am Coll Surg 2015;221:142-52.

(English Language Editor: A. Kassem) 